\title{
Characterization of Glycine Ammonium Sulphate Crystals grown by Slow Evaporation Technique
}

\author{
N.Rathna ${ }^{1}$, V.S.John ${ }^{2}$, T.Chithambarathanu ${ }^{3}$, P.Selvarajan ${ }^{4}$ \\ ${ }^{1}$ Physics Research Centre,S.T.Hindu College,Nagercoil-629002,Tamil Nadu, India \\ ${ }^{2}$ Department of Physics, T.D.M.N.S College,T. Kallikulam-627113,Tamil Nadu, India \\ ${ }^{3}$ Physics Research Centre, S.T.Hindu College, Nagercoil-629003,Tamil Nadu, India. \\ ${ }^{4}$ Department of Physics, Aditanar College of Arts and Science,Tiruchendur-628216, India.
}

\begin{abstract}
The search for new materials possessing high optical nonlinearity is an important task because of their practical applications in harmonic generation, switching and other optical signal processing devices. Some complexes of amino acids with inorganic salts are reported to be promising materials for optical second harmonic generation. An amino acid complex namely glycine ammonium sulphate was synthesized by dissolving glycine and ammonium sulphate in 1:1.5 molar ratio using double distilled water as the solvent by the free evaporation method.The crystal parameters of the grown crystals have been evaluated by single crystal $X R D$ method. The relative Second Harmonic Generation (SHG) efficiency of the grown crystals was measured by Kurtz and Perry Powder technique. Mechanical strength of the grown samples is tested by hardness studies. Thermal studies of the sample were carried out by TG/DTA studies.
\end{abstract}

Keywords: Crystal growth, Dielectric crystal, Microhardness, Single crystal, SHG, TG/DTA, NLO

\section{Introduction}

The search for new materials possessing high optical nonlinearity is an important task because of their practical applications in harmonic generation, switching and other optical signal processing devices. Some complexes of amino acids with inorganic salts are reported to be promising materials for optical second harmonic generation [1-3]. Among the amino acids glycine is the simplest one. Unlike other amino acids it has no asymmetric carbon and is optically inactive. It has three polymorphic crystalline forms of $\alpha, \beta$ and $\gamma$ under ambient conditions. Glycine molecule can exist in zwitterionic form and hence it is capable of forming compounds with anionic, cationic and neutral chemical compounds. Thus a large variety of glycine coordinated compounds can be formed. The $\alpha$ form of glycine crystallizes in centrosymmetric structure hence it does not exhibit second harmonic generation, whereas $\beta$ and $\gamma$ forms crystallizes in noncentrosymmetric crystal structure. The $\beta$ form of glycine is unstable. The $\gamma$-form of glycine exhibits second harmonic generation and the conversion efficiency is reported to be 1.5 times greater than that of KDP [4].

Some complexes of glycine with inorganic salts have already been reported to be promising materials for SHG such as glycine hydrogen phosphate [5], glycine sodium nitrate [6], glycine silver nitrate [7], glycine hydrogen nitrate [8], glycine potassium sulphate[9], glycine lithium sulphate [10], glycine zinc sulphate[11], glycine zinc chloride [12], diglycine manganese chloride[13], triglycine fluoro beryllate [14] etc. In this work, we have made an attempt to combine glycine with ammonium sulphate to form glycine ammonium sulphate (GAS) single crystal. The results obtained are reported herein and discussed.

\section{Crystal Growth}

An aqueous solution of Glycine Ammonium Sulphate (GAS) was prepared by dissolving analytical grade chemicals of glycine and ammonium sulphate in 1:1.5 molar ratio with continuous stirring using a magnetic stirrer for five hours at room temperature. The prepared solution was filtered and kept undisturbed in a constant temperature bath maintained at a temperature of $30{ }^{\circ} \mathrm{C}$. When evaporation takes place slowly, supersaturation is activated. As a result, transparent and colourless single crystals of glycine ammonium sulphate (GAS) were formed at room temperature in a period of about 21 days. 


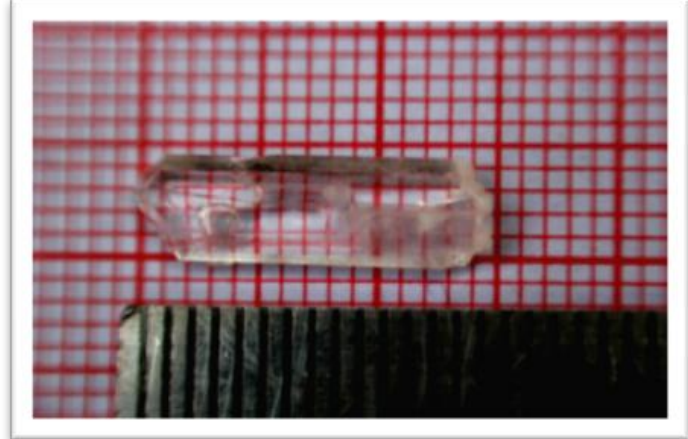

Fig.1: The grown Glycine Ammonium Sulphate (GAS) single crystal

\subsection{Determination of crystal structure}

\section{Results And Discussion}

Single crystal XRD data of GAS crystal were collected from a Single-crystal X-ray diffractometer using ENRAF NONIUS CAD-4 X-ray diffractometer with $\operatorname{MoK}_{\alpha}(\lambda=0.71069 \AA)$ radiation and the obtained data are presented in the table 1 . From the data is observed that the grown crystals crystallize in the monoclinic system with the space group of $\mathrm{P} 2_{1 / \mathrm{C}}$. The density of the crystal is found to be $1.761 \mathrm{~g} / \mathrm{cc}$ and this value was also checked by floatation method.

Table 1: Single Crystal XRD data for GAS crystal

\begin{tabular}{|l|l|}
\hline Unit cell dimensions & $\begin{array}{l}\mathrm{a}=8.262(3) \AA, \alpha=90^{\circ} \\
\mathrm{b}=10.074(2) \AA, \beta=92.66^{\circ} \\
\mathrm{c}=8.632(2) \AA, \gamma=90^{\circ}\end{array}$ \\
\hline Volume of the unit cell & $717.39(4) \AA^{3}$ \\
\hline $\mathrm{Z}$ & 4 \\
\hline Density & $1.761 \mathrm{~g} / \mathrm{cc}$ \\
\hline Wavelength & $0.71069 \AA$ \\
\hline Crystal system, & Monoclinic \\
\hline Space group & $\mathrm{P} 2_{1 / \mathrm{C}}$ \\
\hline
\end{tabular}

\subsection{Second Harmonic Generation}

The NLO property of the grown crystal was tested by passing the output of Nd-YAG Quanta ray laser with fundamental radiation of wavelength $1064 \mathrm{~nm}$ through the crystalline powder GAS sample by Kurtz and Perry method [15]. The emission of green radiation from the sample confirms the second harmonic generation in the crystal. SHG output from the sample was compared with that from KDP crystal. The SHG relative efficiency of GAS crystal is about 0.90 times that of KDP.

\subsection{Microhardness study}

Hardness testing has been widely used to study the strength and deformation in the materials. The method of measuring hardness is not dependent on a single physical property but may involve both the elastic and plastic deformation characteristics such as work hardening coefficient, stiffness constant, yield strength and elastic modulus etc. A good quality crystal was placed on the platform of the Vickers microhardness tester and loads of different magnitudes were applied for a fixed interval of 10 seconds. The hardness number was calculated using the equation $\mathrm{H}_{\mathrm{v}}=1.8544 \mathrm{P} / \mathrm{d}^{2} \mathrm{~kg} / \mathrm{mm}^{2}$ where $\mathrm{P}$ is the applied load in $\mathrm{kg}$ and $\mathrm{d}$ is the diagonal length of the indentation in millimeter. The variation of hardness number with applied load for GAS crystal is shown in the Fig.2. It is observed that Vickers hardness number of the grown crystal increases gradually with increase in load due to the reverse indentation size effect[16].Cracks start develop in the crystal when a load of more than $100 \mathrm{~g}$ is applied,which may be due to the release of internal stress generation with indentation. The relation between load and size of the indentation is given by Mayer's law $P=a^{n}$. Here a and $n$ are constants depending on the material. By plotting $\log P$ versus $\log d$ (Fig. 3.), the value of the work hardening coefficient $n$ was found to be 3.347. Since the value of $\mathrm{n}$ is more than 1.6, the grown crystal is a soft material. 


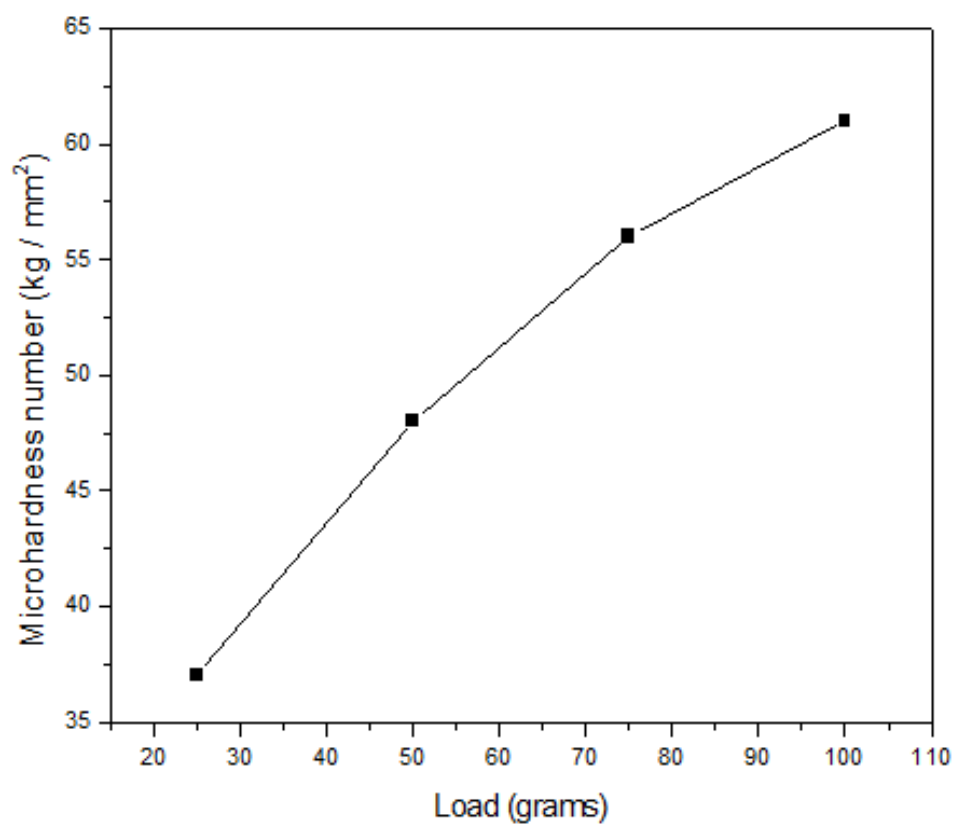

Fig.2: Variation of microhardness number with the applied load for GAS crystal

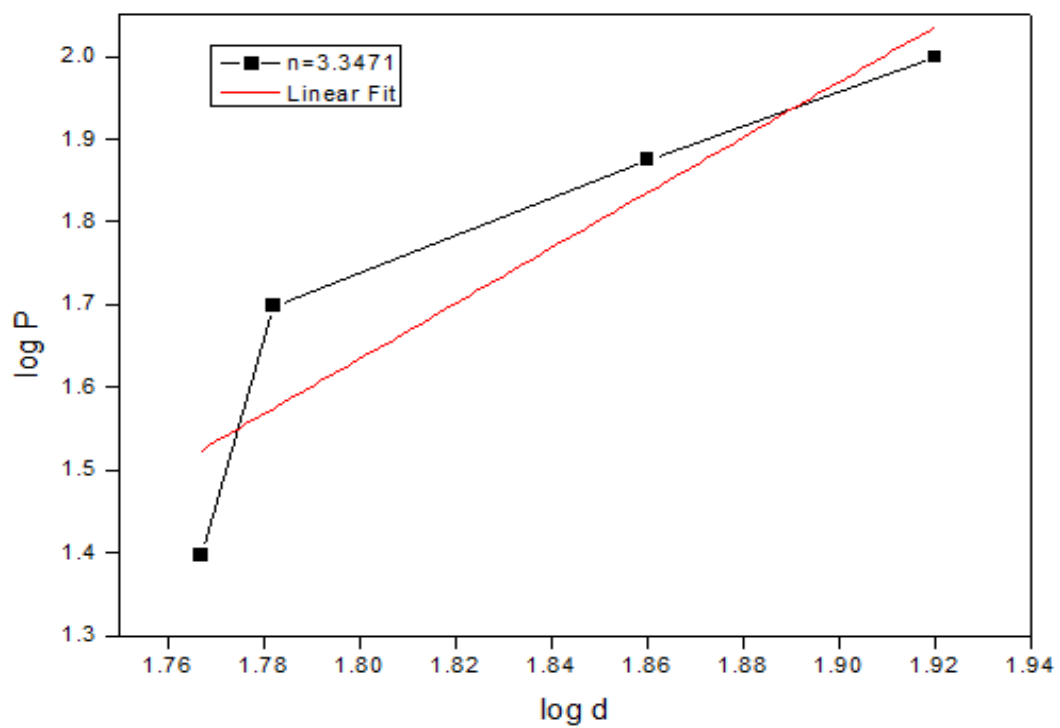

Fig.3. Plot of $\log \mathrm{P}$ versus $\log \mathrm{d}$ for GAS crystal

From the hardness number, the yield strength $\sigma_{y}$ has been calculated using the relation (for $n>2$ ) $\sigma_{y=}$ $\left(\mathrm{H}_{\mathrm{v}} \times(0.1)^{\mathrm{n}-2}\right) / 3$.The calculated values of yield strength $\sigma_{\mathrm{y}}$ for GAS crystal is shown in the Table 2.The elastic stiffness constant $\left(\mathrm{C}_{11}\right)$ has been calculated for the grown crystals using Wooster's empirical relation $\mathrm{C}_{11}=\mathrm{H}_{\mathrm{v}}{ }^{7 / 4}$ $[17,18]$.The obtained values of stiffness constant for different loads are given in the Table 2. It is observed that both the values of yield strength and stiffness constant increase with increase of load in the case of GAS crystal.

Table 2: Yield Strength and Stiffness constant values for GAS crystal

\begin{tabular}{|c|c|c|c|c|}
\hline Grown crystal & Load $(\mathrm{g})$ & $\begin{array}{c}\text { Vicker hardness number } \\
\mathrm{H}_{\mathrm{V}}\left(\mathrm{kg} / \mathrm{mm}^{2}\right)\end{array}$ & $\begin{array}{c}\text { Yield strength } \\
\sigma_{\mathrm{V}}(\mathrm{MPa})\end{array}$ & $\begin{array}{c}\text { Stiffness Constant } \\
\mathrm{C}_{11} \mathrm{x} 10^{14}(\mathrm{~Pa})\end{array}$ \\
\hline \multirow{3}{*}{ GAS } & 25 & 37 & 5.4350 & 9.52793 \\
\cline { 2 - 5 } & 50 & 48 & 7.0509 & 15.0251 \\
\cline { 2 - 5 } & 75 & 56 & 8.2260 & 19.6777 \\
\cline { 2 - 5 } & 100 & 61 & 8.9605 & 22.854 \\
\hline
\end{tabular}




\subsection{Dielectric studies}

Dielectric properties are correlated with electro optic property of the crystals. The dielectric constant is the measure of how easily the material is polarized in an external electric field. The dielectric study on GPS crystal is carried out using the HIOKI 3532-50 LCR HITESTER.Figs.4, 5.show the dielectric constant $\left(\varepsilon_{\mathrm{r}}\right)$ and dielectric loss factors $(\tan \delta$ ) for the GPS crystal. The frequency dependence of the dielectric constant and loss factor for GAS crystals are presented in Figs. 4 and 5. It is observed that the dielectric constant and loss factor have high values at lower frequencies and are low at higher frequencies. It is noticed that these values are found to be increasing with increase of temperature. The dielectric constant of the materials is due to the contribution of electronic, ionic, dipolar or orientation and a space charge polarization which is high relay upon on the frequencies. The low values of dielectric loss of the samples confirm the good quality of dielectric samples. The larger values of dielectric constant at lower frequencies are due to the impedance to the motion of charge carriers at the electrodes, space charge and macroscopic distortion results. According to Miller's rule, the lower values of dielectric constant are suitable for the enhancement of the SHG coefficient [19]. The low dielectric loss at higher frequencies of the sample indicates that the crystals possess less number of electrically active defects and this parameter is of vital importance for nonlinear optical materials in their applications.

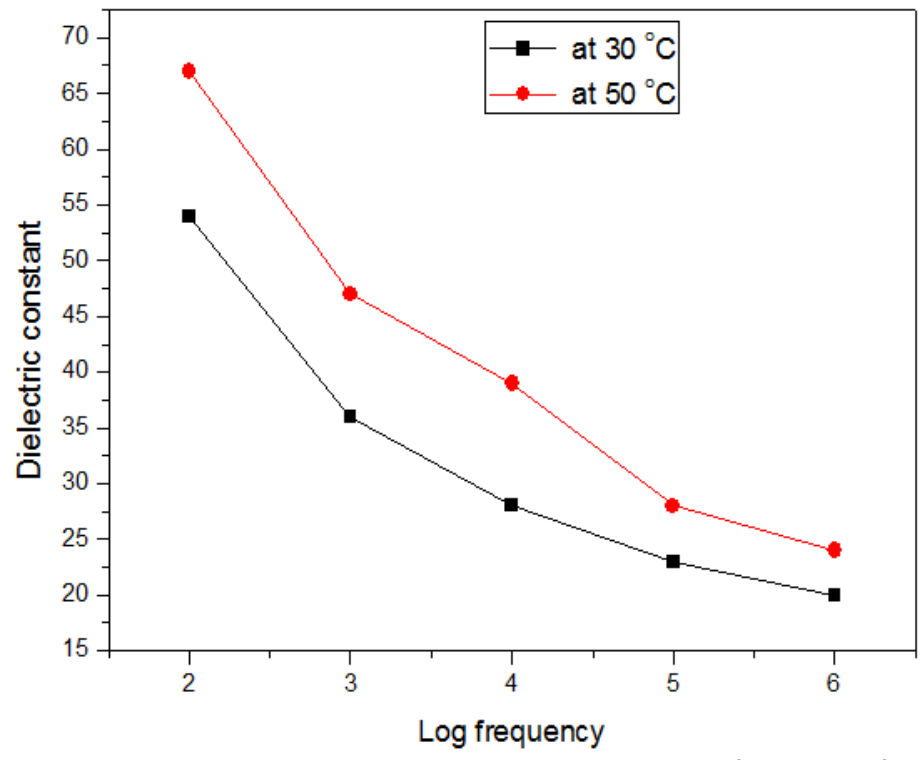

Fig.4: Plot of dielectric constant with frequency at temperatures $30{ }^{\circ} \mathrm{C}$ and $50{ }^{\circ} \mathrm{C}$ for GAS crystal

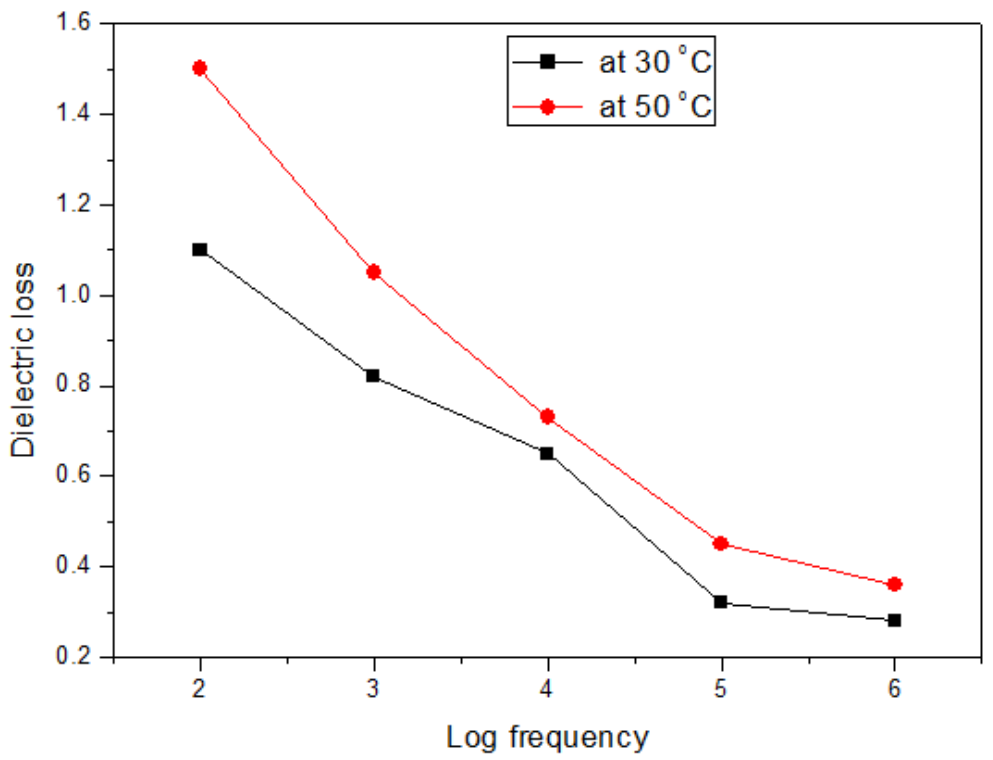

Fig.5: Plot of dielectric loss with frequency at temperatures $30{ }^{\circ} \mathrm{C}$ and $50{ }^{\circ} \mathrm{C}$ for GAS crystal

IV. Conclusion 
Transparent good quality single crystal of Glycine Ammonium Sulphate (GAS) was grown by slow evaporation technique from a mixture of aqueous solution of Glycine and Ammonium Sulphate at constant temperature of $30{ }^{\circ} \mathrm{C}$. The lattice parameters were found by single crystal XRD method. The SHG efficiency of the grown crystal is about 0.9 times that of Potassium dihydrogen Phospahte (KDP). From hardness studies, it is understood that the grown crystal of GAS has moderate hardness and it has reverse indentation size effect. Low dielectric constant and dielectric loss at high frequency suggests that the sample possess enhanced optical quality with lesser defects. Hence it is concluded that optically good quality NLO active Glycine Ammonium Sulphate single crystal with good mechanical stability can be grown by slow evaporation technique and it is suitable for the fabrication of various optoelectronic devices.

\section{Acknowledgements}

The authors would like to thank the staff members who helped to carry out characterization studies from the various research institutions like M.K.University (Madurai), St. Joseph College (Trichy), and Crescent Engineering College (Chennai). Also the authors are thankful to the managements of Aditanar College of Arts and Science, S.T.Hindu college, Nagercoil and T.D.M.N.S College,T. Kallikulam for the encouragement given to us to carry out the research work.

\section{References}

[1]. M. Narayan Bhat, S.M. Dharmaprakash, J. Cryst. Growth 235 (2002) 511.

[2]. A. Deepthy, H.L. Bhat, J. Cryst. Growth 226 (2001) 287.

[3]. R. Rajasekaran, P.M. Ushashree, R. Jayavel, P. Ramasamy, J. Cryst. Growth 229 (2001) 563.

[4]. B. Narayana Moolya, A. Jayarama, M.R. Suresh Kumar, S.M. Dharmaprakash, J. Crystal Growth 280 (2005) 581.

[5]. A.Deepthy and H.L.Bhat, J.Cryst.Growth 226 (2001) 287-293.

[6]. M.N.Bhat and S.M.Dharmaprakash, J.Cryst.Growth 236 (2002)376-380.

[7]. J.K.Mohan Rao and M.A.Vishwamitra, ActaCrystallogr.B28 (1972)1484.

[8]. S.A.Martin Britto and S.Natarajan, Mater.Lett. 62 (2008)2633-2636.

[9]. Ra.Shanmugavadivu, G.Ravi and A.Nixon Azariah, J.Phys. and Chem.Solids 67(2006)1858-1861

[10]. M.R.Suresh Kumar, H.J.Ravindra and S.M.Dharmaprakash, J.Cryst.Growth 306(2007)361-365.

[11]. T.Balakrishnan and K.Ramamurthi, Spectrochim.ActaPart A68 (2007)360-363.

[12]. T.Balakrishnan and K.Ramamurthi, Mater, Lett.62 (2008) 65-68.

[13]. R.Pepinsky, K.Vedam and Y.Okaya, Phys.Rev.110 (1958)1309-1311.

[14]. S.Hosino, T.Mitsui, F.Jona and R.Pepinsky, Phys.Rev.107 (1957)1255-1258.

[15]. S.K.Kurtz and T.T.Perry, J. Appl. Phys.39 (1968) 3798.

[16]. R.Jothi Mani and P.Selvarajan, Int. J. Chem.Tech. Res. 6 (2014) 4702- 4708.

[17]. Wooster W A,Rep Progr Phys,16 (1953) 62.

[18]. Ezhil Vizhi R,Rajan Babu D\&Sahyanarayanan K,Ferroelectrics Lett Sec,37(2)(2010)23.

[19]. A.S.J. Lucia Rose, P. Selvarajan, S. Perumal, Materials Chemistry and Physics 130 (2011) 950- 955. 